\title{
Formation of square lattices in coupled pattern-forming systems
}

\author{
Christopher Strickland*, Daniel A. Pearson ${ }^{\dagger}$, Patrick D. Shipman ${ }^{\ddagger}$ \\ * Department of Mathematics, University of North Carolina-Chapel Hill \\ Chapel Hill, NC, USA \\ * Statistical and Applied Mathematical Sciences Institute \\ Research Triangle Park, NC, USA \\ cstrickland@samsi.edu \\ Departments of Physics ${ }^{\dagger}$ and Mathematics ${ }^{\ddagger}$, Colorado State University \\ Fort Collins, CO, USA \\ dan.pearson@colostate.edu, shipman@math.colostate.edu
}

Received: 5 September 2016, accepted: 18 December 2016, published: 16 January 2017

\begin{abstract}
A wide variety of natural and laboratory systems can produce patterns of ripples, hexagons, or squares. The formation of stable square patterns from partial differential equation models requires specific cubic nonlinearities involving higher-order derivatives. Motivated by plant phyllotaxis, we demonstrate that the coupling of more than one pattern-forming system can produce square patterns without these special nonlinearities.
\end{abstract}

Keywords-pattern formation; phyllotaxis; nanoscale structures

\section{INTRODUCTION: LATTICE PATTERNS IN NATURAL AND LABORATORY SYSTEMS}

Patterns of ripples (Fig. 11 (a)) or hexagons (Fig. 1] (b)) are observed in a wide variety of natural and laboratory systems. Ripples dominate the surface, for example, of the saguaro cactus shown in Fig. 2 (a). They appear as stripes on zebras, on sandy beaches, and in cloud formations. Hexagons, on the other hand, are evident in the surface morphology of the cactus of Fig. 2 (b). Both ripples and hexagons may be observed in RaleighBènard convection experiments [8], the Rosenzweig instability in ferrofluids [12], nanoscale structures formed by bombarding a binary material by a broad ion beam [1], [2], [7], [29], geological formations [17], and landscape-scale vegetation patterns in drylands [4], [6], [18], [19]. Different physical, chemical, or biological mechanisms are at play in these systems, yet remarkably similar patterns form.Fig. 1 shows patterns that result from numerical simulations of the SwiftHohenberg equation [31]

$$
\frac{\partial u}{\partial t}=\alpha u-2 P \nabla^{2} u-\nabla^{4} u+N(u)
$$

where $N(u)$, a nonlinear function of $u$, is chosen to be

$$
N(u)=\beta u^{2}+\gamma u^{3}+\delta \nabla \cdot\left(|\nabla u|^{2} \nabla u\right) .
$$

Depending on choices of coefficients for the nonlinear terms, the surface $u(x, y, \cdot)$ reaches a steady 
state that is a pattern of ripples (Fig 1 (a)), hexagons (Fig 1 (b)), or squares (Fig 1 (c)).
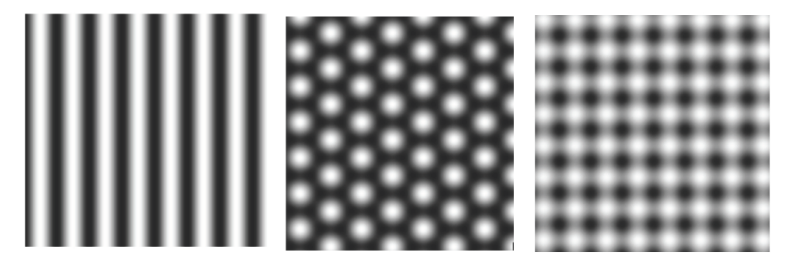

Fig. 1: Gray-scale plots of $u(x, y, t)$ at time $t=$ 10000 resulting from numerical simulations of Eq. (1). The parameter values are $P=1.1, \gamma=10$, and (a) $\beta=0, \delta=0$, (b) $\beta=2, \delta=0$, (c) $\beta=0$, $\delta=1$. The spatial domain is $-60 \leq x, y \leq 60$. (a)

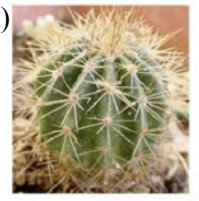

(b)

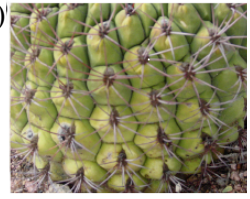

(c)

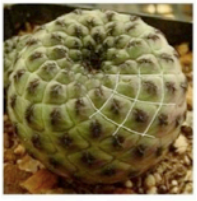

Fig. 2: Cacti displaying planforms of (a) ripples, (b) hexagons, and (c) squares.

The steady-state solutions are approximately given by the sum of only a few Fourier modes. That is, for the case of ripples,

$$
u(x, y, \cdot) \simeq A \mathrm{e}^{i \vec{k} \cdot(x, y)}+\text { c.c. },
$$

where $A$ is a complex amplitude, and c.c. denotes the complex conjugate. For the case of hexagons, there is a triplet $\vec{k}_{1}, \vec{k}_{2}, \vec{k}_{3}$ of wavevectors that are of the same modulus $k_{c}=\left|\vec{k}_{i}\right|, j=1,2,3$ and satisfy the condition $\vec{k}_{1}+\vec{k}_{2}=\vec{k}_{3}$ (see Fig. 3 (a)) such that

$$
u(x, y, \cdot) \simeq \Sigma_{j=1}^{3} A_{j} \mathrm{e}^{i \vec{k}_{j} \cdot(x, y)}+\text { c.c. },
$$

and for the case of squares, there is a pair of orthogonal wavevectors $\vec{k}_{1}, \vec{k}_{2}$ of the same modulus $k_{c}=\left|\vec{k}_{1}\right|=\left|\vec{k}_{2}\right|$ (see Fig. 3 (b)) such that

$$
u(x, y, \cdot) \simeq \Sigma_{j=1}^{2} A_{j} \mathrm{e}^{i \vec{k}_{j} \cdot(x, y)}+\text { c.c. }
$$

Mathematical analysis of Equation (1) proceeds by first performing a linear stability analysis of the homogeneous steady-state solution $u=0$.
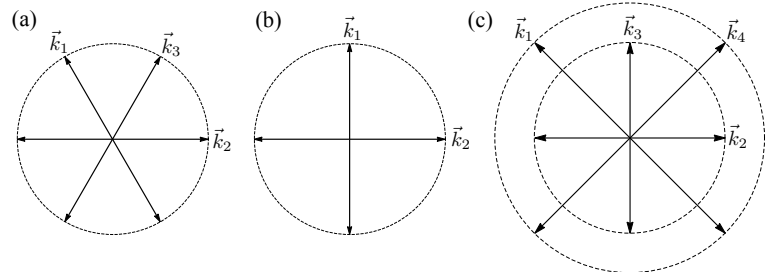

Fig. 3: Wavevectors for Fourier decompositions of (a) hexagon patterns (3) in which there is a triad of wavevectors satisfying $\vec{k}_{1}+\vec{k}_{2}=\vec{k}_{3}$, (b) square patterns (4), and (c) square patterns produced by a coupled pattern-forming system, in which there are overlapping triads of wavevectors satisfying $\vec{k}_{1}+$ $\vec{k}_{2}=\vec{k}_{3}$ and $\vec{k}_{2}+\vec{k}_{3}=\vec{k}_{4}$.

This determines the modulus $k_{c}$ of wavevectors that will be present in the pattern. All Fourier modes $A(t) \mathrm{e}^{i \vec{k} \cdot(x, y)}$ with wavevector $\vec{k}$ on a circle of radius $k_{c}$ are linearly unstable so that their amplitudes $A(t)$ grow in time for $t \simeq 0$. Once the amplitudes become large enough, nonlinear functions of these amplitudes become large enough to dampen the growth and to allow for interactions between the modes that determine the resulting steady-state pattern [5], [14].

While there is an abundance of examples of ripple and hexagon patterns in laboratory and natural systems, square patterns are relatively rare. As one example, in [13] it is shown that a cubic nonlinearity of the form $\delta \nabla \cdot\left(|\nabla u|^{2} \nabla u\right)$ in the equations of motion describing the surface evolution of a crystalline material being bombarded by a broad ion beam results in a pattern of squares. We propose in this paper an alternative to cubic terms involving higher-order derivatives, namely the coupling of two pattern-forming systems, that can also result in a pattern of squares.

The idea that coupled mechanisms may result in square patterns comes to us from observations of patterns on plants. Section 2 describes these plant patterns and a model for plant pattern formation proposed in [23] that couples biochemical and biomechanical mechanisms. Although we suggest in [23] that this model can produce square patterns, this paper gives the first numerical and analyti- 


\section{Strickland et al., Formation of square lattices in coupled ...}

cal evidence that this is the case by numerical simulations and analysis of a simplified system given in Section 3 that captures the key features of the original model. Numerical simulations of the simplified system produce square patterns for certain choices of parameters. The results agree with linear stability analysis and weakly nonlinear analysis of the simplified system, as given in the Appendix.

\section{Phyllotaxis AND A SyStem OF COUPLED PATTERN-FORMING EQUATIONS}

Phyllotaxis refers to the arrangement (taxis) on plants of leaves (phylla) or their analogs such as bracts on a pine cone, seeds in a seedhead, or spines on a cactus. We describe in Section II.A how a pattern of squares underlies many of these phyllotactic patterns. In Section II.B, we review a systems of coupled PDEs, proposed in [23] as a model for the formation of phyllotactic patterns on plants.

A. Phyllotactic planforms and the Fibonacci sequence
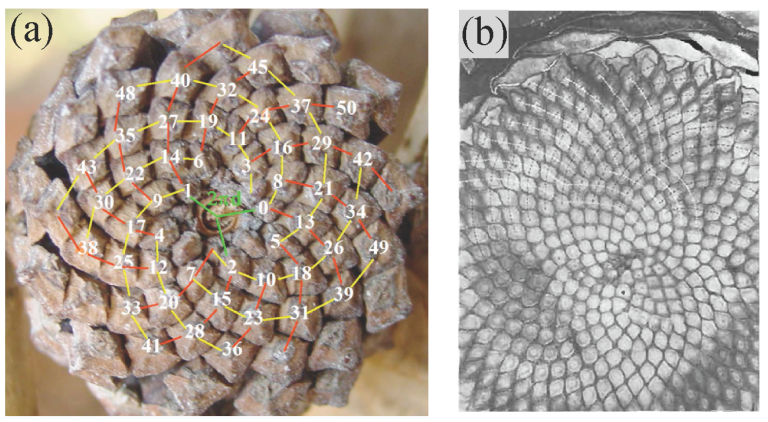

Fig. 4: (a) A pinecone with the bracts numbered in order of their distance to the center. Also marked are eight counterclockwise spirals (in yellow) and thirteen clockwise spirals (in red) formed by connecting adjacent bracts. (b) From [3], a sunflower seed head with clockwise and counterclockwise spirals marked. The spiral families are different near the outer boundary of the seed head compared to the center.

The square bracts on the pinecone shown in Fig. 4 (a) have been numbered in sequence of their distance from the center of the cone. Connecting bracts that have adjacent sides results in the eight (yellow) counterclockwise spirals and thirteen (red) clockwise spirals. The numbers of spirals in the clockwise and counterclockwise spiral families are called the parastichy numbers for the pattern. The pair of parastichy numbers 8 and 13 is not unusual. Indeed, the spiral numbers observed on plants are typically consecutive members of the Fibonacci sequence $\left\{F_{j}\right\}=$ $1,1,2,3,5,8,13,21, \ldots$.

The parastichy numbers may vary even within one plant's pattern. This is illustrated by the sunflower seed heads of Fig. 4 (b) and Fig. 5. The transition between spiral numbers in the Fibonacci sequence can, in fact, be continuous. To understand this, consider the function

$$
w(r, \sigma)=\sum_{j=1}^{N} \hat{A}\left(\frac{r}{F_{j}}\right) \cos \left(\vec{k}_{j}(r) \cdot \vec{x}\right),
$$

of polar coordinates, radius $r$ and angle $\sigma$. In (5), $\vec{x}=(r, \sigma)$, and the amplitudes $\hat{A}(\rho)=\frac{\rho^{2}}{\frac{1}{5} \rho^{4}+2}$ and wavevectors $\vec{k}_{j}=\left(l_{j}(r), \frac{F_{j}}{r}\right)$ depend on the radius $r$. We give phenomenological derivations of these forms of $\hat{A}(\rho)$ and the radial wavenumber $l_{j}(r)$ in [22], [28] to illustrate how parastichy numbers may change continuously in spiral phyllotaxis; we do not claim that these are the functions observed on plants. In [24], we numerically calculate a function $\hat{A}(\rho)$ from phyllotactic patterns produced by a PDE model. Figs. 5 and 6 show plots of Equation (5) for $F_{j}$ in the Fibonacci sequence. The bottom panels of Fig. 6 show the graph of the function $\hat{A}\left(\frac{r}{F_{j}}\right)$ for various values of the radius $r$, namely $r_{1}, r_{2}=\frac{\phi}{4} r_{1}, r_{3}=\frac{\phi}{2} r_{1}, r_{4}=\frac{3 \phi}{4} r_{1}$, and $r_{5}=\phi r_{1}$, as marked in the figure. A selfsimilarity in the pattern becomes apparent: The vector of amplitudes for $r=r_{1}$ is equal to the vector of amplitudes for $r=r_{5}=\phi r_{1}$ after shifting the $F_{j}$. That is, writing $A_{j}(r) \doteq \hat{A}\left(\frac{r}{j}\right)$, $\left(\ldots, A_{8}\left(r_{1}\right), A_{13}\left(r_{1}\right), A_{21}\left(r_{1}\right), A_{34}\left(r_{1}\right), \ldots\right)=$ $\left(\ldots, A_{13}\left(\phi r_{1}\right), A_{21}\left(\phi r_{1}\right), A_{34}\left(\phi r_{1}\right), A_{55}\left(\phi r_{1}\right), \ldots\right)$.

Also note that, at $r=r_{1}, A_{8}=A_{34}<A_{13}=$ $A_{21}$, and at $r=r_{5}, A_{13}=A_{55}<A_{21}=A_{34}$. 


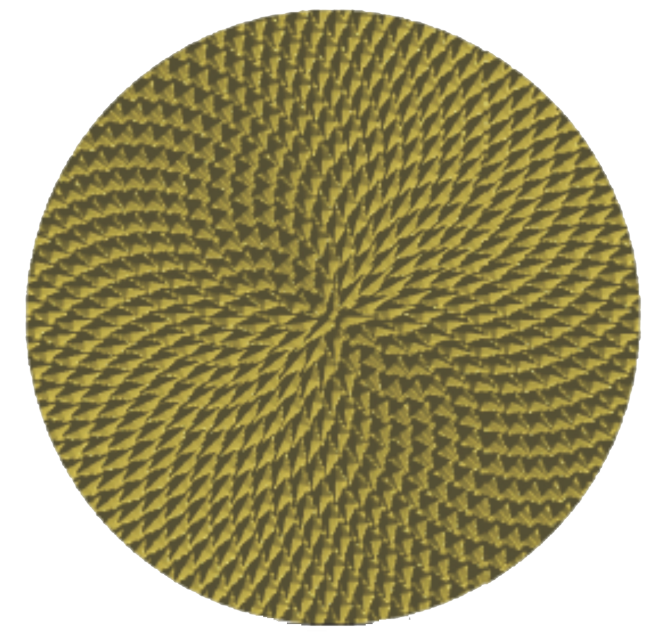

Fig. 5: Plot of the function given by Equation (5), replicating a sunflower seed head.

Recalling that written in terms of the index $j$, $F_{6}=8, F_{7}=13, F_{8}=21, F_{9}=34$, and $F_{10}=55$, we also note a corresponding relationship of wavevectors: at $r=r_{1}, \vec{k}_{6}+\vec{k}_{7}=\vec{k}_{8}$, and $\vec{k}_{7}+\vec{k}_{8}=\vec{k}_{9}$, whereas at $r=r_{2}, \vec{k}_{7}+\vec{k}_{8}=\vec{k}_{9}$, and $\vec{k}_{8}+\vec{k}_{9}=\vec{k}_{10}$.

The key observation is that the square patterns that are evident at radii $r=r_{1}$ and $r=r_{5}$ are formed not by only two Fourier modes with wavevectors as in Fig. 3 (b), but by overlapping triads of modes that satisfy summation relations similar to the wavevectors of Fig. 3 (a) that produce hexagons. As depicted in Fig. 3 (c), in order for this to occur, two of the vectors are larger in modulus than the other two. These are the wavevectors corresponding to the smaller amplitudes. For example, at $r=r_{1}$, the wavevectors $\vec{k}_{6}, \vec{k}_{9}$ corresponding to the smaller amplitudes $A_{8}=A_{34}$ are longer than the wavevectors $\vec{k}_{7}, \vec{k}_{8}$ corresponding to the larger amplitudes $A_{13}=A_{21}$.

\section{B. A mechanistic model for phyllotactic patterns}

In this section, we review a system of partial differential equation that has been proposed as a model for the formation of phyllotactic patterns and that incorporates biochemical and biophysical mechanisms, each of which could produce a pattern on its own.
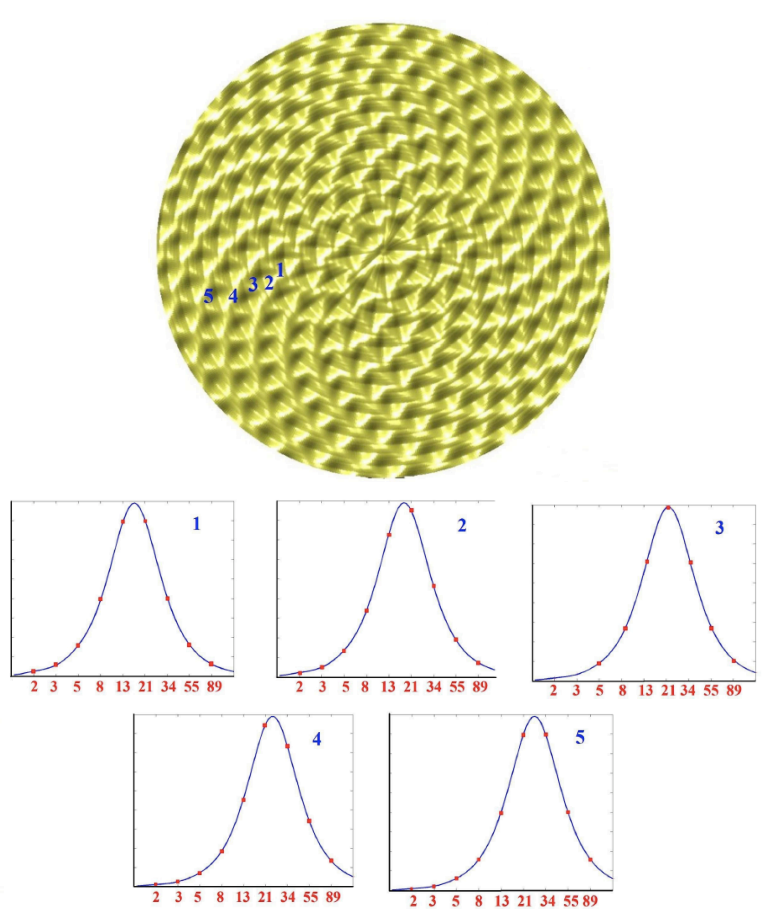

Fig. 6: Top: Graph of the function (5). Bottom: Graphs of $\hat{A}\left(\rho=r / m_{j}\right)$ as a function of $m_{j}$ for various values of $r, r=r_{1}, r_{2}=\frac{\phi}{4} r_{1}, r_{3}=$ $\frac{\phi}{2} r_{1}, r_{4}=\frac{3 \phi}{4} r_{1}$, and $r_{5}=\phi r_{1}$.

Growth of a shoot tip or formation of flowers occurs in regions of active cell growth and division at apical meristems. A schematic diagram of a shoot apical meristem (SAM) is shown in Fig. 7 . Small bumps called primordia on the plant surface, which will become leaves, form not at the very center of the SAM, but in an annular region which we call the generative region and which is marked as Region 2 in Fig. 7 .

What mechanisms lead to the formation of primordia in the generative region? There is evidence for both biomechanical and biochemical mechanisms which may interact with each other.

The idea of the biomechanical mechanism is as follows: If the outer skin (the tunica) of the plant is growing more quickly than the inner tissue, then a compressive stress will build up in the tunica. If that compressive stress increases above a large enough threshold, then the tunica will become 
unstable and buckle under the stress. Biologist Paul Green proposed in the 2000's that primordia are the result of this buckling [9]-[11].

The plant hormone auxin influences cell growth, and other experiments suggest that auxin itself may be spatially patterned in the generative region, with primordia forming where there is a higher auxin concentration [15], [16]. Auxin is produced uniformly throughout the generative region, but the key idea of the groups of Kuhlemeier and Meyerowitz [16], [30] is that PIN1 proteins in cell walls transport auxin from cells with lower concentrations of auxin to cells with higher auxin concentrations. This produces an instability that allows for pattern formation.

The biomechanical and biochemical mechanisms may interact in that stress states can impact the action of PIN1 proteins. In [23], we incorporate both mechanisms into a mathematical model of three partial differential equations for the tunica surface deformation $w(x, y, t)$, a potential $F(x, y, t)$ for the stresses in the tunica, and the auxin concentration difference $g(x, y, t)$ from a mean auxin concentration.

We refer the reader to [23] for a complete description of this model, which reads, in nondimensionalized parameters,

$$
\begin{array}{r}
\zeta_{m} w_{t}+\Delta^{2} w+P \Delta w+\kappa w+\gamma w^{3} \\
+C \Delta F-[F, w]=0, \\
\Delta^{2} F+\Delta g-C \Delta w+\frac{1}{2}[w, w]=0, \\
\zeta_{g} g_{t}+D_{g} \Delta^{2} g+H \Delta g+d g+\delta g^{3}+\kappa_{1} \nabla(g \nabla g) \\
+\kappa_{2} \nabla\left(\nabla g \nabla^{2} g\right)-b \Delta F=0,
\end{array}
$$

where $[f, g]=f_{x x} g_{y y}+f_{y y} g_{x x}-2 f_{x y} g_{x y}$. Equations (6a) and (6b) are the Föppl-von Kárman equations for a thin elastic to which a compressive stress has been applied. Equation (6c) is a continuum approximation of a spatially discrete model proposed in [16] for auxin transport.

Both the mechanical system 6a, 6b and the auxin system (6c) may produce an instability of the homogeneous state $w=F=g=0$ to a pattern. For the mechanical system, this instability

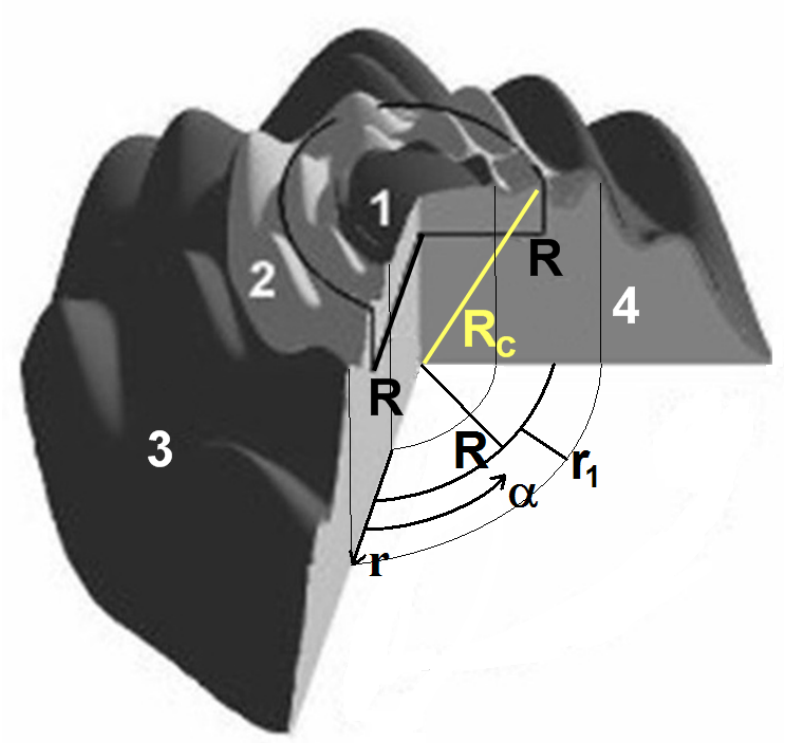

Fig. 7: A schematic of the plant shoot apical meristem (SAM). Cells form but primorida do not form at the center of the SAM (Region 1). Region 2 is the annular generative region where primoria form. In Region 3, no new primordia form, but there is active cell generation and differentiation.

occurs if the compressive stress, expressed in the nondimensional parameter $P$, exceeds a critical value. For the auxin system, this instability occurs if the relative magnitude of auxin transport compared to auxin diffusion, expressed in the nondimensional parameter $H$, exceeds a critical value. If both the mechanical instability and the auxin instability are active, the possibly different natural wavelengths of the patterns that would result from either instability alone allow for differences in phyllotactic configurations (the underlying lattice) and the surface deformation. In [23] we analyze a variety of scenarios in which the elastic and auxin instabilities may cooperate or compete.

\section{SQUARE PATTERN FORMATION IN COUPLED EQUATIONS OF SWIFT-HOHENBERG TYPE}

Equations (6a) and (6c) are both of SwiftHohenberg type (1) with the addition of nonlinear terms that couple the equations. We expect therefore for the following simpler and more analyti- 
cally tractable system of coupled Swift-Hohenberg equations for two fields $u(x, y, t)$ and $v(x, y, t)$ to produce similar steady-state patterns:

$$
\begin{gathered}
u_{t}+\Delta^{2} u+2 P \Delta u+u+\alpha_{1} v \\
\quad+\beta_{1} u^{2}+\beta_{3} u v+\beta_{5} v^{2}+\gamma_{1} u^{3}=0 \\
v_{t}+\Delta^{2} v+2 H \Delta v+L v+\alpha_{2} u \\
\quad+\beta_{2} v^{2}+\beta_{4} v u+\beta_{6} u^{2}+\gamma_{2} v^{3}=0 .
\end{gathered}
$$

This system has the uniform steady-state solution $u(x, y, t)=v(x, y, t)=0$. Linear stability analysis, given in the Appendix, reveals that in the absence of linear coupling $\left(\alpha_{1}=\alpha_{2}=0\right)$, $u(x, y, t)=0$ is stable for $P \leq P_{c}=1$, but unstable to Fourier modes with wavevectors of modulus close to $|k|=1$ for $P>P_{c}=1$. Similarly, $v(x, y, t)=0$ is stable for $H \leq H_{c}=\sqrt{L}$, but unstable to Fourier modes with wavevectors of modulus close to $|k|=\sqrt[4]{L}$ for $H>H_{c}=\sqrt{L}$. The modified conditions for instabilities in the presence of linear coupling are given the the Appendix, but the key point is that the parameter $L$ determines how the the wavelength of the pattern favored by the equation for $v$ compares to that for $u$. If $L=1$, then both equations would yield patterns of the same natural wavelength, but if $L$ is larger (smaller) than 1 , then the wavelength of the pattern favored by the equation for $v$ will be smaller (larger) than that favored by the equation for $u$.

If $P=P_{c}+\epsilon \chi$ and $H=H_{c}+\epsilon \chi$, where $\chi \sim O(1)$, are slightly above their respective bifurcation thresholds (as measured by the small parameter $\epsilon$ ), Fourier modes with moduli close to 1 and $\sqrt[4]{L}$ grow in amplitude with time and interact via the nonlinear terms in the equations. The wavevectors for these modes are depicted as the two circles in Fig. 3 (c). In the Appendix, we demonstrate a weakly nonlinear asymptotic analysis that allows us to derive a system of ordinary differential equations for the amplitudes of these excited Fourier modes. This analysis begins with an Ansatz for the form of the solution, namely

$$
\begin{aligned}
& u=\varepsilon \sum_{j=1}^{N}\left(A_{j} e^{i \vec{k}_{j} \vec{x}}+A_{j}^{*} e^{-i \vec{k}_{j} \vec{x}}\right)+\varepsilon^{2} u_{1}+\varepsilon^{3} u_{2}+\ldots \\
& v=\varepsilon \sum_{j=1}^{N}\left(B_{j} e^{i \vec{k}_{j} \vec{x}}+B_{j}^{*} e^{-i \vec{k}_{j} \vec{x}}\right)+\varepsilon^{2} v_{1}+\varepsilon^{3} v_{2}+\ldots
\end{aligned}
$$

Here, $N$ is the number of interacting modes in the Fourier expansion of the order- $\epsilon$ term, $A_{j}=A_{j}(T=\varepsilon t), B_{j}=B_{j}(T=\varepsilon t)$, and $\varepsilon^{2} u_{1}+\varepsilon^{3} u_{2}+\ldots$, and $\varepsilon^{2} v_{1}+\varepsilon^{3} v_{2}+\ldots$ are the correction terms. A condition for solvability of the correction terms leads to a set of ordinary differential equations for the time-evolution of the complex amplitudes $A_{j}(t)$ and $B_{j}(t)$.

The result of a numerical simulation of Equation (7) for a choice of parameter values that includes nonlinear coupling terms but not linear coupling $\left(\alpha_{1}=\alpha_{2}=0\right)$ is shown in Fig. 8. The initial conditions are low-amplitude white noise. We employ a Fourier spectral method with periodic boundary conditions and a fourth-order exponential time differencing Runge-Kutta method for the time stepping as the numerical technique, and the spatial grid is $256 \times 256$.

The Fourier transform of the pattern shown in Fig. 8 (a) is shown in Fig. 8 (b). There are two circles of excited wavevectors, as marked in Fig. 8 (c). The reason for this is the parameter choice $L=4.7$ in (7), which allows for the wavevectors of modulus $\sqrt[4]{4.7} \simeq 1.47$ to be excited by the field $v$, while wavevectors of length 1 are excited by the field $u$ (see the linear stability analysis in the Appendix). These wavevectors interact via nonlinear terms in Equations (7), and a discrete set of Fourier modes with wavevectors $\vec{k}_{1}, \ldots, \vec{k}_{4}$ such that $\vec{k}_{1}+\vec{k}_{2}=\vec{k}_{3}$ and $\vec{k}_{2}+\vec{k}_{3}=\vec{k}_{4}$, as marked in Fig. 8 (c) (which may be compared to Fig. 3 (c)), dominates the pattern.

Motivated by the results of the numerical simulation, we carry out in the Appendix the weakly nonlinear asymptotic analysis, choosing in Equation (8) $N=4$ and the overlapping triad conditions $\vec{k}_{1}+\vec{k}_{2}=\vec{k}_{3}$ and $\vec{k}_{2}+\vec{k}_{3}=\vec{k}_{4}$. This results in a system of eight ordinary differential equations for the amplitudes $A_{j}, B_{j}, j=1, \ldots, 4$ of the Fourier modes in (8). This is the system (15). A numerical simulation of (15) for the parameter values of the simulation in Fig. 8 is shown in Fig. 9 . The amplitudes reach a steady state in which $A_{2}=A_{3}=B_{2}=B_{3}$ and $A_{1}=A_{4}=B_{1}=B_{4}$. This is consistent with the Fourier spectrum shown 


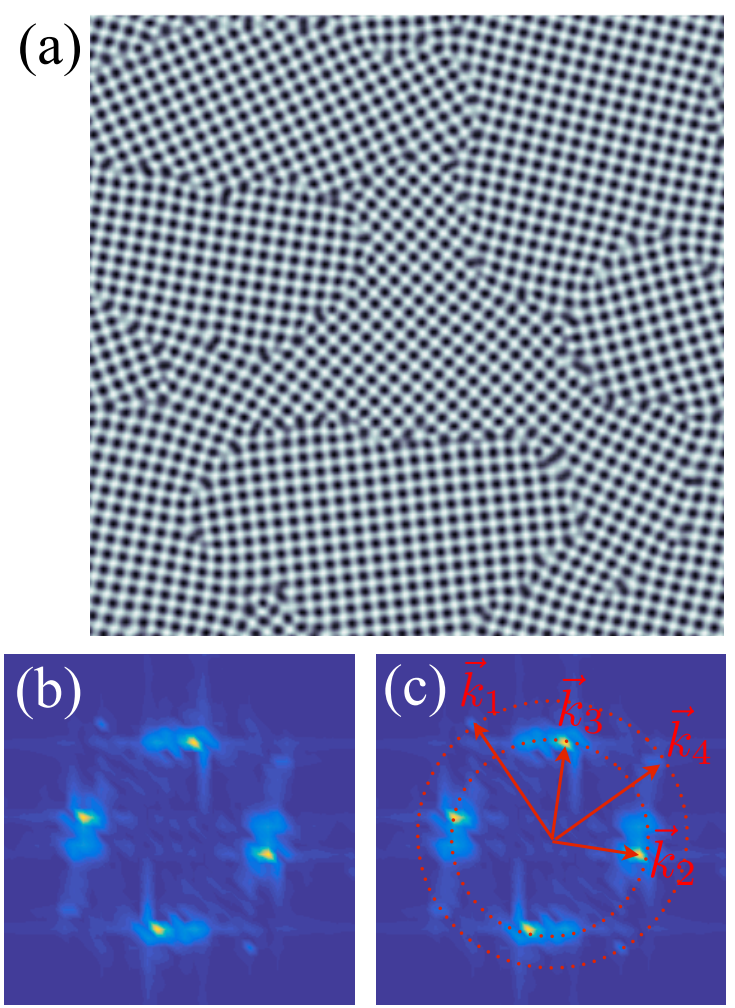

Fig. 8: (a) Gray-scale plot of $u(x, y, t)$ at time $t=$ 25000 , resulting from numerical simulations of the system (7). The parameter values are $P=1.1$, $H=2.2, L=4.7, \alpha_{1}=\alpha_{2}=0, \beta_{1}=-3, \beta_{2}=$ $3, \beta_{3}=\beta_{4}=1$, and $\beta_{5}=\beta_{6}=-5$. The spatial domain is $-120 \leq x, y \leq 120$. (b,c) the Fourier transform of a portion of the surface $u(x, y, t)$ at time $t=25000$. The domain in wavevector $\vec{k}=\left(k_{x}, k_{y}\right)$-space is $-0.8 \leq k_{x}, k_{y} \leq 0.8$. The wavevectors $\vec{k}_{1}, \ldots, \vec{k}_{4}$ are marked in panel (c).

in Fig. $8(b, c)$, in which the modes on the circle of larger radius have smaller amplitude. It is also consistent with the motivation given in Section II.A of patterns observed in plant phyllotaxis.

Other possible patterns and their stability would be found by a bifurcation analysis of the amplitude equations (15). The relevance of this analysis to plant phyllotaxis would require that the length scale of the patterns produced by the system (6) are in accord with those observed on plants. In [23], we provide suggestions on experiments to

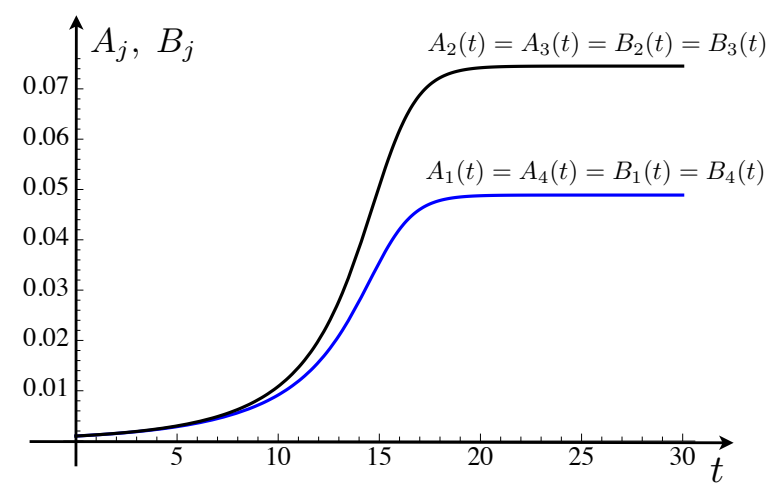

Fig. 9: Numerical solution of the amplitude equations (15) for the parameter values $P=1.1$, $H=2.2, L=4.7, \alpha_{1}=\alpha_{2}=0, \beta_{1}=-3$, $\beta_{2}=3, \beta_{3}=\beta_{4}=1$, and $\beta_{5}=\beta_{6}=-5$.

determine some of the parameter values relevant to plants. Although motivated by phyllotaxis in this paper, the framework of coupled patternforming systems is relevant to other phenomena. This includes nanoscale pattern formation induced by bombarding a binary alloy by a broad ion beam [1], [2], [7], [29]. In these experiments, collision cascades induced by the ions hitting the surface of the alloy result in the sputtering of material from the surface. Curvature-dependent sputter yield and phase separation are two independent pattern-forming mechanisms that may both contribute to the observed pattern. Squares can also appear in Turing patterns: Recently, Li and colleauges form patterns of squares in simulations of coupled reaction-diffusion equations [20].

\section{ACKNOWLEDGMENTS}

We thank R. Mark Bradley and Alan C. Newell for valuable discussions. We are also grateful to the National Science Foundation for its support through Grant No. DMS-1022635 to P. D. S. and DMR-1305449 to R. M. Bradley and P. D. S.

\section{APPENDIX: LINEAR AND NONLINEAR ANALYSIS}

In this Appendix, we provide the details of the linear stability analysis and the nonlinear analysis 


\section{Strickland et al., Formation of square lattices in coupled ...}

(the derivation of the nonlinear amplitude equations) for the system (7).

\section{Linear stability analysis}

The system (7) has the uniform steady-state solution $u=u_{s}=0, v=v_{s}=0$. We will examine the stability of this solution by introducing a small perturbation of this solution and determining if the perturbation has linear growth or decay. Write the perturbation as

$$
\begin{aligned}
& u=u_{s}+\hat{u} e^{i \vec{k} \vec{x}} e^{\sigma t} \\
& v=v_{s}+\hat{v} e^{i \vec{k} \vec{x}} e^{\sigma t},
\end{aligned}
$$

where $\vec{k}=\left(k_{x}, k_{y}\right)$, and $\hat{u}$ and $\hat{v}$ are constants. Inserting (9) into the linearization of Equation (7), we obtain

$\sigma\left(\begin{array}{l}\hat{u} \\ \hat{v}\end{array}\right)=\left(\begin{array}{cc}-k^{4}+2 P k^{2}-1 & -\alpha_{1} \\ -\alpha_{2} & -k^{4}+2 H k^{2}-L\end{array}\right)\left(\begin{array}{l}\hat{u} \\ \hat{v}\end{array}\right)$,

where $k^{2}=k_{x}^{2}+k_{y}^{2}$. This vector equation implies that $\sigma$ is an eigenvalue of the matrix in Equation (10). The two solutions for $\sigma$ are

$\sigma_{ \pm}(\vec{k})=\frac{\sigma_{u}(\vec{k})+\sigma_{v}(\vec{k})}{2} \pm \sqrt{\left(\frac{\sigma_{u}(\vec{k})-\sigma_{v}(\vec{k})}{2}\right)^{2}+\alpha_{1} \alpha_{2}}$,

where $\sigma_{u}(k) \doteq-k^{4}+2 P k^{2}-1$ and $\sigma_{v}(k) \doteq$ $-k^{4}+2 H k^{2}-L$. We will use $\sigma_{+}(\vec{k})$ to denote the larger of the two eigenvalues.

Note that if $\hat{v}=0$, we have only the equation $\sigma \hat{u}=-k^{4} \hat{u}+2 P k^{2} \hat{u}-\hat{u}$ which reduces to $\sigma=$ $-k^{4}+2 P k^{2}-1=\sigma_{u}(k)$ after dividing through by $\hat{u}$. Similarly, $\sigma=\sigma_{v}(\vec{k})$ when $u=0$. The coupling terms alter these values, and for positive $\alpha_{1} \alpha_{2}, \sigma_{+}(\vec{k})$ is always larger than either $\sigma_{u}(\vec{k})$ or $\sigma_{v}(\vec{k})$. Since $\sigma$ is the growth rate for the system and instabilities occur when $\sigma>0$, the coupling terms actually help create an instability. The choice of $\sigma_{+}$is non-trivial for $L \neq 1$ even in the case where there is no linear coupling $\left(\alpha_{1}=\alpha_{2}=0\right)$; $\sigma_{+}=\sigma_{u}(\vec{k})$ for some values of $\vec{k}$ and $\sigma_{+}=\sigma_{v}(\vec{k})$ for others. By definition, we always choose the larger of the two values.

In the case of no linear coupling $\left(\alpha_{1}=\alpha_{2}=0\right)$, we have the following: For $P<1, \sigma_{u}(\vec{k})<0$ for all $\vec{k}$
For $P=1, \sigma_{u}(1)=0$ and $\sigma_{u}(\vec{k})<0$ for all $k \neq 1$.

For $P>1, \sigma_{u}(\vec{k})>0$ for certain values of $\vec{k}$ inside a finite band centered around $|k|=1$.

Similarly,

For $H<\sqrt{L}, \sigma_{v}(\vec{k})<0$ for all $\vec{k}$.

For $H=\sqrt{L}, \sigma_{v}(\sqrt[4]{L})$ and $\sigma_{v}(\vec{k})<0$ for all $k \neq \sqrt[4]{L}$.

For $H>\sqrt{L}, \sigma_{v}(\vec{k})>0$ for certain values of $\vec{k}$ inside a finite band centered around $|k|=\sqrt[4]{L}$.

The parameters $P$ and $H$ thus serve as bifurcation parameters with respective bifurcation values $P=P_{c r} \doteq 1$ and $H=H_{c r} \doteq \sqrt{L}$. In the absence of linear coupling, the uniform steadystate solution is linearly stable for $P<P_{c r}$ and $H<H_{c r}$. Perturbations with certain wavevectors are unstable for $P>P_{c r}$ and $H>H_{c r}$. Recall that for general values of $\alpha_{1}$ and $\alpha_{2}$ (with $\alpha_{1} \alpha_{2}>0$ ), the modified value of $\sigma_{+}(\vec{k})$ will be greater than either $\sigma_{u}$ or $\sigma_{v}$ (say $\sigma^{*}$ for the general case).

We identify the set of active wavevectors, $\vec{k}$, to be those for which $\sigma_{+}(k)>-\sigma^{*}$ for some small $-\sigma *<0$. The goal is to analyze the cases where $\sigma_{+}$is just above zero, when the active modes begin to interact through the nonlinear terms in the equations.

\section{Nonlinear Analysis}

For $\sigma_{+}$above the critical value of $\sigma_{+}=0$, there are active modes, and these will interact through the nonlinear terms in (7). We derive evolution equations for the amplitudes of the active modes by asymptotically expanding the solutions $u$ and $v$ with respect to a small parameter $\varepsilon$ measuring how far $\sigma_{+}$is above 0 . That is, we assume that the bifurcation parameters are close to their respective critical values: for $\chi \sim O(1)$,

$$
\begin{gathered}
P=P_{c r}+\varepsilon \chi=1+\varepsilon \chi \\
H=H_{c r}+\varepsilon \chi=\sqrt{L}+\varepsilon \chi
\end{gathered}
$$

We also assume that the coefficients of the cubic terms are of order $\epsilon^{-1}$ :

$$
\varepsilon \gamma_{1,2}=\hat{\gamma}_{1,2}, \hat{\gamma}_{i} \sim O(1)
$$




\section{Strickland et al., Formation of square lattices in coupled ...}

As an Ansatz for the form of the solution to (7), we assume that $u$ and $v$ are an order- $\varepsilon$ perturbation from the steady state solution plus correction terms that are of higher order in $\varepsilon$ :

$u=\varepsilon \sum_{j=1}^{N}\left(A_{j} e^{i \vec{k}_{j} \vec{x}}+A_{j}^{*} e^{-i \vec{k}_{j} \vec{x}}\right)+\varepsilon^{2} u_{1}+\varepsilon^{3} u_{2}+\ldots$

$v=\varepsilon \sum_{j=1}^{N}\left(B_{j} e^{i \vec{k}_{j} \vec{x}}+B_{j}^{*} e^{-i \vec{k}_{j} \vec{x}}\right)+\varepsilon^{2} v_{1}+\varepsilon^{3} v_{2}+\ldots$

Here, $N$ is the number of interacting modes in the Fourier expansion of the order- $\epsilon$ term, $A_{j}=$ $A_{j}(T=\varepsilon t), B_{j}=B_{j}(T=\varepsilon t)$, and $\varepsilon^{2} u_{1}+\varepsilon^{3} u_{2}+$ $\ldots$, and $\varepsilon^{2} v_{1}+\varepsilon^{3} v_{2}+\ldots$ are the correction terms. Inserting (13) into the system (7) and collecting coefficients of powers of $\varepsilon$ yields, at order $\varepsilon$ simply the expression $0=0$, but at order $\varepsilon^{2}$, we obtain the relations

$$
\begin{aligned}
& \left(\Delta^{2}+2 P_{c r} \Delta+1\right) u_{1}= \\
& \quad 2 \chi \sum_{j=1}^{N} A_{j} e^{i \vec{k}_{j} \vec{x}}+A_{j}^{*} e^{-i \vec{k}_{j} \vec{x}} \\
& \quad-\sum_{j=1}^{N}\left(\frac{A_{j}}{d T} e^{i \vec{k}_{j} \vec{x}}+\frac{A_{j}^{*}}{d T} e^{-i \vec{k}_{j} \vec{x}}\right) \\
& \quad-\alpha_{1} \sum_{j=1}^{N}\left(B_{j} e^{i \vec{k}_{j} \vec{x}}+B_{j}^{*} e^{-i \vec{k}_{j} \vec{x}}\right) \\
& \quad-\beta_{1}\left(\sum_{j=1}^{N} A_{j} e^{i \vec{k}_{j} \vec{x}}+A_{j}^{*} e^{-i \vec{k}_{j} \vec{x}}\right)^{2} \\
& \quad-\beta_{3}\left(\sum_{j=1}^{N} A_{j} e^{i \vec{k}_{j} \vec{x}}+A_{j}^{*} e^{-i \vec{k}_{j} \vec{x}}\right) \\
& \quad \times\left(\sum_{j=1}^{N} B_{j} e^{i \vec{k}_{j} \vec{x}}+B_{j}^{*} e^{-i \vec{k}_{j} \vec{x}}\right) \\
& \quad-\beta_{5}\left(\sum_{j=1}^{N} B_{j} e^{i \vec{k}_{j} \vec{x}}+B_{j}^{*} e^{-i \vec{k}_{j} \vec{x}}\right)^{2} \\
& \quad-\hat{\gamma}_{1}\left(\sum_{j=1}^{N} A_{j} e^{i \vec{k}_{j} \vec{x}}+A_{j}^{*} e^{-i \vec{k}_{j} \vec{x}}\right)^{3} \\
& \left(\Delta^{2}+2 H_{c r} \Delta+L\right) v_{1}= \\
& \quad 2 \chi \sum_{j=1}^{N} B_{j} e^{i \vec{k}_{j} \vec{x}}+B_{j}^{*} e^{-i \vec{k}_{j} \vec{x}} \\
& \quad-\sum_{j=1}^{N}\left(\frac{B_{j}}{d T} e^{i \vec{k}_{j} \vec{x}}+\frac{B_{j}^{*}}{d T} e^{-i \vec{k}_{j} \vec{x}}\right) \\
& \quad-\alpha_{2} \sum_{j=1}^{N}\left(A_{j} e^{i \vec{k}_{j} \vec{x}}+A_{j}^{*} e^{-i \vec{k}_{j} \vec{x}}\right) \\
& \quad-\beta_{2}\left(\sum_{j=1}^{N} B_{j} e^{i \vec{k}_{j} \vec{x}}+B_{j}^{*} e^{-i \vec{k}_{j} \vec{x}}\right)^{2} \\
& \quad-\beta_{4}\left(\sum_{j=1}^{N} A_{j} e^{i \vec{k}_{j} \vec{x}}+A_{j}^{*} e^{-i \vec{k}_{j} \vec{x}}\right) \\
& \quad \times\left(\sum_{j=1}^{N} B_{j} e^{i \vec{k}_{j} \vec{x}}+B_{j}^{*} e^{-i \vec{k}_{j} \vec{x}}\right) \\
& \quad-\beta_{6}\left(\sum_{j=1}^{N} A_{j} e^{i \vec{k}_{j} \vec{x}}+A_{j}^{*} e^{-i \vec{k}_{j} \vec{x}}\right)^{2} \\
& \quad-\hat{\gamma}_{2}\left(\sum_{j=1}^{N} B_{j} e^{i \vec{k}_{j} \vec{x}}+B_{j}^{*} e^{-i \vec{k}_{j} \vec{x}}\right)^{3}
\end{aligned}
$$

The first of these equations has the form $\left(\Delta^{2}+\right.$ $\left.2 P_{c r} \Delta+1\right) u_{1}=C e^{i \vec{k} \vec{x}}$ where $P_{c r}=1$ and $|\vec{k}|=1$ (recall that this value was found to be critical for instabilities in the linear case with no coupling when $P \geq 0$ ). This equation has the form $u_{1}=D e^{i \vec{k} \vec{x}}$. Thus, if the coefficient of $e^{i \overrightarrow{k_{j}} \vec{x}}$ on the right-hand side of (14) is nonzero, resonance allows the solution to grow without bound, and our asymptotic expansion is invalid since the correction terms are no longer small. Our solvability condition is therefore that the coefficient of $e^{i \vec{k}_{j} \vec{x}}$ on the right-hand side of (14) be zero.

We now examine the case of (14) for $N=4$, where $\vec{k}_{1}+\vec{k}_{2}=\vec{k}_{3}$, and $\vec{k}_{2}+\vec{k}_{3}=\vec{k}_{4}$. Requiring that the coefficients of the $e^{i \vec{k}_{j} \vec{x}}$ terms, $j=1 \ldots 4$ sum to zero results in a set of eight differential equations for the time evolution of the amplitudes $A_{j}$ and $B_{j}, j=1 \ldots 4$ which read

$$
\begin{aligned}
& \frac{d A_{1}}{d T}=2 \chi A_{1}-\alpha_{1} B_{1}-\beta_{1} A_{2}^{*} A_{3}-\beta_{3}\left(A_{2}^{*} B_{3}+A_{3} B_{2}^{*}\right) \\
& -\beta_{5} B_{2}^{*} B_{3}-3 \hat{\gamma}_{1} A_{1}\left(2 \sum_{j=1}^{4}\left|A_{j}\right|^{2}-\left|A_{1}\right|^{2}\right) \\
& \frac{d A_{2}}{d T}=2 \chi A_{2}-\alpha_{1} B_{2}-\beta_{1}\left(A_{1}^{*} A_{3}+A_{3}^{*} A_{4}\right) \\
& -\beta_{3}\left(A_{1}^{*} B_{3}+A_{3} B_{1}^{*}+A_{4} B_{2}^{*}+A_{2}^{*} B_{4}\right) \\
& -\beta_{5}\left(B_{1}^{*} B_{3}+B_{3}^{*} B_{4}\right) \\
& -3 \hat{\gamma}_{1} A_{2}\left(2 \sum_{j=1}^{4}\left|A_{j}\right|^{2}-\left|A_{2}\right|^{2}\right) \\
& \frac{d A_{3}}{d T}=2 \chi A_{3}-\alpha_{1} B_{3}-\beta_{1}\left(A_{1} A_{2}+A_{2}^{*} A_{4}\right) \\
& -\beta_{3}\left(A_{1} B_{2}+A_{2} B_{1}+A_{2}^{*} B_{4}+A_{4} B_{2}^{*}\right) \\
& -\beta_{5}\left(B_{1} B_{2}+B_{2}^{*} B_{4}\right) \\
& -3 \hat{\gamma}_{1} A_{3}\left(2 \sum_{j=1}^{4}\left|A_{j}\right|^{2}-\left|A_{3}\right|^{2}\right) \\
& \frac{d A_{4}}{d T}=2 \chi A_{4}-\alpha_{1} B_{4}-\beta_{1} A_{2} A_{3}-\beta_{3}\left(A_{2} B_{3}+A_{3} B_{2}\right) \\
& -\beta_{5} B_{2} B_{3}-3 \hat{\gamma}_{1} A_{4}\left(2 \sum_{j=1}^{4}\left|A_{j}\right|^{2}-\left|A_{4}\right|^{2}\right) \\
& \frac{d B_{1}}{d T}=2 \chi B_{1}-\alpha_{2} A_{1}-\beta_{2} B_{2}^{*} B_{3}-\beta_{4}\left(B_{2}^{*} A_{3}+B_{3} A_{2}^{*}\right) \\
& -\beta_{6} A_{2}^{*} A_{3}-3 \hat{\gamma}_{2} B_{1}\left(2 \sum_{j=1}^{4}\left|B_{j}\right|^{2}-\left|B_{1}\right|^{2}\right) \\
& \frac{d B_{2}}{d T}=2 \chi B_{2}-\alpha_{2} A_{2}-\beta_{2}\left(B_{1}^{*} B_{3}+B_{3}^{*} B_{4}\right) \\
& -\beta_{4}\left(B_{1}^{*} A_{3}+B_{3} A_{1}^{*}+B_{4} A_{2}^{*}+B_{2}^{*} A_{4}\right) \\
& -\beta_{6}\left(A_{1}^{*} A_{3}+A_{3}^{*} A_{4}\right) \\
& -3 \hat{\gamma}_{2} B_{2}\left(2 \sum_{j=1}^{4}\left|B_{j}\right|^{2}-\left|B_{2}\right|^{2}\right) \\
& \frac{d B_{3}}{d T}=2 \chi B_{3}-\alpha_{2} A_{3}-\beta_{2}\left(B_{1} B_{2}+B_{2}^{*} B_{4}\right) \\
& -\beta_{4}\left(B_{1} A_{2}+B_{2} A_{1}+B_{2}^{*} A_{4}+B_{4} A_{2}^{*}\right) \\
& -\beta_{6}\left(A_{1} A_{2}+A_{2}^{*} A_{4}\right) \\
& -3 \hat{\gamma}_{1} B_{3}\left(2 \sum_{j=1}^{4}\left|B_{j}\right|^{2}-\left|B_{3}\right|^{2}\right) \\
& \frac{d B_{4}}{d T}=2 \chi B_{4}-\alpha_{2} A_{4}-\beta_{2} B_{2} B_{3}-\beta_{4}\left(B_{2} A_{3}+B_{3} A_{2}\right) \\
& -\beta_{5} A_{2} A_{3}-3 \hat{\gamma}_{2} B_{4}\left(2 \sum_{j=1}^{4}\left|B_{j}\right|^{2}-\left|B_{4}\right|^{2}\right) \text {. }
\end{aligned}
$$

Now observe that for $k^{2}=1$ 


$$
\begin{aligned}
\sigma_{u}(\vec{k}) & =-k^{4}+2 P k^{2}-1 \\
& =-k^{4}+2(1+\varepsilon \chi) k^{2}-1 \\
& =-k^{4}+2 k^{2}-1+2 \varepsilon \chi k^{2} \\
& =2 \varepsilon \chi .
\end{aligned}
$$

We obtain a similar relation for $\sigma_{v}(\vec{k})$. This allows us to rewrite the linear terms in the amplitude equations as $2 \chi A_{i}=\varepsilon^{-1} \sigma_{u}(\vec{k}) A_{i}$ and $2 \chi B_{i}=$ $\varepsilon^{-1} \sigma_{v}(\vec{k}) B_{i}$.

Recalling that $T=\varepsilon t$, we rescale $A_{i}$ and $B_{i}$ as

$$
\begin{array}{ll}
\hat{A}_{i}=\varepsilon A_{i} & \frac{d \hat{A}_{i}}{d t}=\frac{d A_{i}}{d T} \frac{d T}{d t}=\frac{d A_{i}}{d T} \varepsilon \\
\hat{B}_{i}=\varepsilon B_{i} & \frac{d \hat{B}_{i}}{d t}=\frac{d B_{i}}{d T} \frac{d T}{d t}=\frac{d B_{i}}{d T} \varepsilon \\
\hat{\alpha_{i}}=\varepsilon \alpha_{i} &
\end{array}
$$

Using these and the earlier rescaling $\hat{\gamma}_{1,2}=\varepsilon \gamma_{1,2}$, we obtain, after multiplying through by $\varepsilon^{2}$, the following set of differential equations (the hat notation has been suppressed on all $A_{i}$ and $B_{i}$ ):

$$
\begin{aligned}
\frac{d A_{1}}{d t}=\sigma_{u} & (\vec{k}) A_{1}-\alpha_{1} B_{1}-\beta_{1} A_{2}^{*} A_{3} \\
& -\beta_{3}\left(A_{2}^{*} B_{3}+A_{3} B_{2}^{*}\right)-\beta_{5} B_{2}^{*} B_{3} \\
& -3 \gamma_{1} A_{1}\left(2 \sum_{j=1}^{4}\left|A_{j}\right|^{2}-\left|A_{1}\right|^{2}\right) \\
\frac{d A_{2}}{d t}=\sigma_{u}(\vec{k}) A_{2}-\alpha_{1} B_{2}-\beta_{1}\left(A_{1}^{*} A_{3}+A_{3}^{*} A_{4}\right) & -\beta_{3}\left(A_{1}^{*} B_{3}+A_{3} B_{1}^{*}+A_{4} B_{2}^{*}+A_{2}^{*} B_{4}\right) \\
& -\beta_{5}\left(B_{1}^{*} B_{3}+B_{3}^{*} B_{4}\right) \\
& -3 \gamma_{1} A_{2}\left(2 \sum_{j=1}^{4}\left|A_{j}\right|^{2}-\left|A_{2}\right|^{2}\right) \\
\frac{d A_{3}}{d t}=\sigma_{u}(\vec{k}) A_{3}-\alpha_{1} B_{3}-\beta_{1}\left(A_{1} A_{2}+A_{2}^{*} A_{4}\right) & -\beta_{3}\left(A_{1} B_{2}+A_{2} B_{1}+A_{2}^{*} B_{4}+A_{4} B_{2}^{*}\right) \\
& -\beta_{5}\left(B_{1} B_{2}+B_{2}^{*} B_{4}\right) \\
& -3 \gamma_{1} A_{3}\left(2 \sum_{j=1}^{4}\left|A_{j}\right|^{2}-\left|A_{3}\right|^{2}\right) \\
\frac{d A_{4}}{d t}= & \sigma_{u}(\vec{k}) A_{4}-\alpha_{1} B_{4}-\beta_{1} A_{2} A_{3} \\
& -\beta_{3}\left(A_{2} B_{3}+A_{3} B_{2}\right)-\beta_{5} B_{2} B_{3} \\
& -3 \gamma_{1} A_{4}\left(2 \sum_{j=1}^{4}\left|A_{j}\right|^{2}-\left|A_{4}\right|^{2}\right)
\end{aligned}
$$

$$
\begin{aligned}
\frac{d B_{1}}{d t}=\sigma_{v} & (\vec{k}) B_{1}-\alpha_{2} A_{1}-\beta_{2} B_{2}^{*} B_{3} \\
& -\beta_{4}\left(B_{2}^{*} A_{3}+B_{3} A_{2}^{*}\right)-\beta_{6} A_{2}^{*} A_{3} \\
& -3 \gamma_{2} B_{1}\left(2 \sum_{j=1}^{4}\left|B_{j}\right|^{2}-\left|B_{1}\right|^{2}\right) \\
\frac{d B_{2}}{d t}=\sigma_{v} & (\vec{k}) B_{2}-\alpha_{2} A_{2}-\beta_{2}\left(B_{1}^{*} B_{3}+B_{3}^{*} B_{4}\right) \\
& -\beta_{4}\left(B_{1}^{*} A_{3}+B_{3} A_{1}^{*}+B_{4} A_{2}^{*}+B_{2}^{*} A_{4}\right) \\
& -\beta_{6}\left(A_{1}^{*} A_{3}+A_{3}^{*} A_{4}\right) \\
& -3 \gamma_{2} B_{2}\left(2 \sum_{j=1}^{4}\left|B_{j}\right|^{2}-\left|B_{2}\right|^{2}\right)
\end{aligned}
$$

$$
\begin{aligned}
\frac{d B_{3}}{d t}=\sigma_{v} & (\vec{k}) B_{3}-\alpha_{2} A_{3}-\beta_{2}\left(B_{1} B_{2}+B_{2}^{*} B_{4}\right) \\
& -\beta_{4}\left(B_{1} A_{2}+B_{2} A_{1}+B_{2}^{*} A_{4}+B_{4} A_{2}^{*}\right) \\
& -\beta_{6}\left(A_{1} A_{2}+A_{2}^{*} A_{4}\right) \\
& -3 \gamma_{2} B_{3}\left(2 \sum_{j=1}^{4}\left|B_{j}\right|^{2}-\left|B_{3}\right|^{2}\right)
\end{aligned}
$$

$$
\begin{aligned}
\frac{d B_{4}}{d t}=\sigma_{v} & (\vec{k}) B_{4}-\alpha_{2} A_{4}-\beta_{2} B_{2} B_{3} \\
& -\beta_{4}\left(B_{2} A_{3}+B_{3} A_{2}\right)-\beta_{6} A_{2} A_{3} \\
& -3 \gamma_{2} B_{4}\left(2 \sum_{j=1}^{4}\left|B_{j}\right|^{2}-\left|B_{4}\right|^{2}\right) .
\end{aligned}
$$

\section{REFERENCES}

[1] R. M. Bradley and P. D. Shipman, Spontaneous Pattern Formation Induced by Ion Bombardment of Binary Compounds. Phys. Rev. Lett. 105 (2010) 145501. http://journals.aps.org/prl/abstract/10.1103/PhysRevLett. 105.145501

[2] R. M. Bradley and P. D. Shipman, A Surface Layer of Altered Composition Can Play a Key Role in Nanoscale Pattern Formation Induced By Ion Bombardment. Appl. Surf. Sci. 258 (2012) 4161.

http://www.sciencedirect.com/science/article/pii/ S0169433211010385

[3] A. H. Church, On the Relation of Phyllotaxis to Mechanical Laws, Williams and Norgate, 1904.

[4] P. Couteron, and O. Lejeune, Periodic spotted patterns in semi-arid vegetation explained by a propagationinhibition model. J. of Ecology 89 (2001) 616628. http://onlinelibrary.wiley.com/doi/10.1046/j.0022-0477. 2001.00588.x/abstract

[5] M. Cross, H. Greenside, Pattern Formation and Dynamics in Nonequilibrium Systems, Cambridge UP, 2009.

[6] V. Deblauwe, P. Couteron, O. Lejeune, J. Bogaert, N. Barbier, Environmental modulation of self-organized periodic vegetation patterns in Sudan. Ecography 34 (2011) 9901001.

http://onlinelibrary.wiley.com/doi/10.1111/j.1600-0587. 2010.06694.x/abstract

[7] S. Facsko, T. Dekorsy, C. Koerdt, C. Trappe, H. Kurz, A. Vogt, and H. L. Hartnagel, Formation of ordered nanoscale semiconductor dots by ion sputtering. Science 285 (1999) 1551.

http://science.sciencemag.org/content/285/5433/1551 
[8] A.V. Getling, Rayleigh-Bénard Convection: Structures and Dynamics, World Scientific, 1998.

[9] C. R. Goodall, P. B. Green, Quantitative analysis of surface growth. Botanical Gazette 147 (1) (1986) 1-15. http://www.journals.uchicago.edu/doi/abs/10.1086/ 337562

[10] P. B. Green, Expression of pattern in plants: Combining molecular and calculus-based biophysical paradigms. Am. J. Botany 86 (8) (1999) 1059-1076. http://www.amjbot.org/content/86/8/1059.full

[11] P. B. Green, C. R. Steele, S. C. Rennich, Phyllotactic patterns: a biophysical mechanism for their origin. Ann. Bot. 77 (5) (1996) 515-528. http://aob.oxfordjournals.org/content/77/5/515

[12] C. Gollwitzer, I. Rehberg, R. Richter, Via hexagons to squares in ferrofluids: experiments on hysteretic surface transformations under variation of the normal magnetic field. Journal of Physics: Condensed Matter 18 (2006). http://iopscience.iop.org/article/10.1088/0953-8984/18/ 38/S08/meta

[13] B. Hashmi, P. D. Shipman and R. M. Bradley, Highly Ordered Square Arrays of Nanoscale Pyramids Produced by Ion Bombardment of a Crystalline Binary Material. Phys. Rev. E 93 (2016) 032207.

http://journals.aps.org/pre/abstract/10.1103/PhysRevE.93. 032207

[14] R. Hoyle, Pattern Formation: An Introduction to Methods. Cambridge UP, Cambridge, 2006.

[15] M. G. Heisler, O. Hamant, P. Krupinski, M. Uttewaal, C. Ohno, H. Jönsson, J. Traas, E. M. Meyerowitz, Alignment between PIN1 polarity and microtubule orientation in the shoot apical meristem reveals a tight coupling between morphogenesis and auxin transport. PLOS Biology (2011).

http://journals.plos.org/plosbiology/article?id=10.1371/ journal.pbio.1000516

[16] H. Jönsson, M. Heisler, B. E. Shapiro, E. M. Meyerowitz, E. Mjolsness, An auxin-driven polarized transport model for phyllotaxis, PNAS 103 (5) (2006) 16331638. http://www.pnas.org/content/103/5/1633.full

[17] W. B. Krantz, K. J. Gleason, N. Caine, Patterned ground. Scientific American 259, 6 (1988).

[18] R. Lefever, O. Lejeune, On the origin of tiger bush, Bulletin of Mathematical Biology 59 (1997) 263294. http://link.springer.com/article/10.1007/BF02462004

[19] R. Lefever, N. Barbier, P. Couteron, O. Lejeune, Deeply gapped vegetation patterns: On crown/root allometry, criticality, and desertification, Journal of Theoretical Biology 261 (2009) 194209.

http://www.sciencedirect.com/science/journal/00225193/ 261/2

[20] J. Li, H. Wang, Q. Ouyang, Square Turing patterns in reaction-diffusion equations with coupled layers. Chaos 24 (2014) $1-5$.

http://scitation.aip.org/content/aip/journal/chaos/24/2/10. 1063/1.4875262
[21] A. C. Newell, P. D. Shipman, Plants and Fibonacci. J. Stat. Phys. 121 (2005) 5-6. http://link.springer.com/article/10.1007/ s10955-005-8665-7

[22] A. C. Newell, P. D. Shipman, A new invariant in plant phyllotaxis. Analysis and Applications 6 (4) (2008) 383399.

[23] A. C. Newell, P. D. Shipman, Z. Sun, Phyllotaxis: cooperation and competition between mechanical and biochemical processes, Journal of Theoretical Biology 251 (2008) 3. http://www.worldscientific.com/doi/abs/10.1142/ S0219530508001237

[24] M. Pennybacker, P. D. Shipman, A. C. Newell, Phyllotaxis: Some progress, but a problem far from over. Physica D 306 (2016) 48-81.

http://www.sciencedirect.com/science/article/pii/ S0167278915000743

[25] W. Pesch, L. Kramer, Nonlinear analysis of spatial structures in two-dimensional anisotropic pattern forming systems. Z. Phys. B 63 (1986) 121. http://link.springer.com/article/10.1007/BF01312586

[26] P. D. Shipman, A. C. Newell, Phyllotactic patterns on plants. Phys. Rev. Lett. 92 (2004) 16. http://journals.aps.org/prl/abstract/10.1103/PhysRevLett. 92.168102

[27] P. D. Shipman, A. C. Newell, Polygonal planforms and phyllotaxis on plants. J. Theor. Biol. 236 (2005) 154-197.

http://www.sciencedirect.com/science/article/pii/ S0022519305000950

[28] P. D. Shipman, Discrete and continuous invariance in phyllotactic tilings. Phys. Rev. E 81 (2010) id. 031905. http://journals.aps.org/pre/pdf/10.1103/PhysRevE.81. 031905

[29] P. D. Shipman and R. M. Bradley, Theory of nanoscale pattern formation induced by normal-incidence ion bombardment of binary compounds. Phys. Rev. B 84 (2011) 085420.

http://journals.aps.org/prb/abstract/10.1103/PhysRevB. 90.085428

[30] R. S. Smith, S. Guyomarc'h, T. Mandel, D. Reinhardt, C. Kuhlemeier, P. Prusinkiewicz, A plausible model for phyllotaxis, PNAS, 103 (5) (2006) 1301-1306. http://www.pnas.org/content/103/5/1301.full

[31] J. Swift, P. C. Hohenberg, Hydrodynamic fluctuations at the convective instability. Phys. Rev. A 15 (1977) 319-328.

http://journals.aps.org/pra/abstract/10.1103/PhysRevA. 15.319

[32] R. F. Williams, The Shoot Apex and Leaf Growth, Cambridge UP, Cambridge, 1975. 